\title{
Eva-Maria Seng
}

\section{EINLEITUNG}

Ein besonders niedriger Wasserstand im Zürichsee im Winter 1853/54 führte zur Entdeckung der Pfahlbauten. Weitere Fundstätten wurden nach intensiver Suche in anderen Alpenrandseen auch im Bodensee aufgetan. Badische und württembergische Altertumsforscher bargen damals zahlreiche Funde aus dem Schlamm des Bodensees und weiterer Seen und Moore des Voralpenlandes und Oberschwabens. Ein wahres Pfahlbaufieber erfasste die Schweiz und den deutschen Südwesten und führte zu zahlreichen Grabungen, der Sammlung von Pfahlbaufunden in den damals neu aufkommenden Urund Frühgeschichtlichen Museen und Sammlungen bis hin zur Präsentation von Pfahlbaufunden und Rekonstruktionsmodellen auf den Weltausstellungen in Paris 1867 und Wien 1873. Das Phänomen der Pfahlbauten war auch eine der Initialzündungen zur Einrichtung Ur- und Frühgeschichtlicher Forschungsinstitute an Universitäten des deutschen Südwestens wie auch der staatlichen archäologischen Denkmalpflege in Baden und Württemberg. Die sehr guten Erhaltungsbedingungen im Moor und unter Wasser unter Sauerstoffabschluss, konservierten organische Materialien wie Holz, Textilien etc. auf einzigartige Weise und ermöglichen damit einzigartige Erkenntnisse über die neolithischen und bronzezeitlichen Kulturen vom 5. bis 1. Jahrtausend vor Christus. Die Funde und Realien dieser frühen Kulturen ohne schriftliche Überlieferung lassen Aussagen zu Wirtschaftsweise, Hausbau, Ernährung, Kleidung, Landwirtschaft und All$\operatorname{tag} z u$.

Trotz aller Faszination, welche die Funde dieser Pfahlbau- und Feuchtbodensiedlungen auslösten, war von Beginn an ein Problem deren Erschließung und Vermittlung an eine interessierte Öffentlichkeit. Im 19. und frühen 20. Jahrhundert beschäftigte die Forschung insbesondere die Bauweise und Konstruktion der Häuser und die Anlage der Siedlungen. Dies führte zu Rekonstruktionszeichnungen und zur Erstellung von Modellen wie schon kurz nach der Entdeckung der Funde nach Vorlagen des Schweizer Altertumsforschers und Nestors der Pfahlbauforschung Ferdinand Keller, der maßgeblich die Theorie über die Entstehung und das Alter der Funde bis hin zur Namensgebung „Pfahlbauten“ prägte. Im 20. Jahrhundert entstanden Nachbauten wie im 1922 eröffneten Pfahlbaumuseum in Unteruhldingen. Nach einer ideologischen Vereinnahmung der Ur- 
und Frühgeschichte wie auch insbesondere der Pfahlbauforschung durch die Nationalsozialisten im Rahmen des Reichsbundes für Deutsche Vorgeschichte wurde erst in den 1970er Jahren wieder eine eigentliche Pfahlbauforschung oder Feuchtbodenarchäologie durch das Landesdenkmalamt Baden-Württemberg initiiert und mit der Einrichtung der Arbeitsstelle für das feuchte Kulturgut 1981 in Hemmenhofen am Bodensee auch administrativ verankert. Neue Forschungsergebnisse ermöglichten die interdisziplinäre Zusammenarbeit von naturwissenschaftlichen Spezialisten mit Archäologen. In den Forschungsstellen in Hemmenhofen und der Schweiz sind Archäobotaniker, Archäozoologen, Geologen und Dendrologen mit Feuchtboden- und Unterwasserarchäologen in einer Forschergruppe verbunden, die neue umwelt- und klimageschichtliche Fragestellungen in Verbindung mit sozialen und kulturellen Gesellschaft- und Wirtschaftsentwicklungen einerseits und deren Langzeitstrukturen andererseits erlauben.

Auf Initiative der Schweiz wurde anläßlich des 150-jährigen Jubiläums der Entdeckung der Pfahlbauten seit 2004 ein Antrag für 111 prähistorische Stätten in der Schweiz, Deutschland, Italien, Österreich, Frankreich und Slowenien erarbeitet, die 2011 Aufnahme in die UNESCO-Welterbeliste fanden. Die beteiligten Stätten und Staaten verpflichteten sich damit auch zum Austausch und zur Abstimmung ihrer Aktivitäten sowie zur regelmäßigen Abhaltung von Konferenzen. Nach Tagungen über Archäologie und Erosion trat insbesondere das Thema des Transfers wissenschaftlicher Ergebnisse an die Öffentlichkeit und damit die Vermittlung des unsichtbaren Welterbes in den Mittelpunkt des Interesses der neuen Welterbestätten.

Im Jahr 2014 wurde deshalb in Konstanz vom Landesamt für Denkmalpflege Baden-Württemberg und dem Bayerischen Landesamt für Denkmalpflege in Zusammenarbeit mit dem Lehrstuhl für Materielles und Immaterielles Kulturerbe der Universität Paderborn eine dreitägige Tagung der Internationalen Koordinierungsgruppe World Heritage Prehistoric Pile Dwellings around the Alps zum Thema "Erschließung und Vermittlung des Welterbes ,Prähistorische Pfahlbauten um die Alpen'“ abgehalten. Die Veranstalter kooperierten mit der Universität Konstanz, der Internationalen Bodenseekonferenz, der Deutschen UNESCO-Kommission, der Swiss Coordination Group und der Arbeitsgemeinschaft Prähistorische Pfahlbauten um die Alpen Baden-Württemberg. Im Mittelpunkt der Fragestellung standen auf der Tagung neben den Pfahlbauten als unsichtbares Welterbe auch der Vergleich mit anderen seriellen transnationalen Welterbestätten wie dem Limes, dem Wattenmeer, dem Loire -Tal und die dort gemachten Erfahrungen mit Kulturtourismus und den unterschiedlichen Formen der Vermittlung wie der experimentellen Archäologie, der Welterbebildung in den UNESCO-Projektschulen oder durch Filme und öffentliche Medien.

Der vorliegende Band gibt die Beiträge der Tagung wieder, ergänzt um eine Schilderung der Entstehungsgeschichte der Pfahlbausammlung im Rosgartenmuseum in Konstanz durch dessen heutigen Direktor Tobias Engelsing. Daniel Gutscher entwickelt in seinem Beitrag aus dem UNESCO-Gründungsdokument von 1945 und der darin formulierten bildungspolitischen Ziele wie auch der UNESCO-Konvention zum Schutz des 
Kultur- und Naturerbes von 1972 einen Bildungsauftrag für Welterbestätten im einzelnen und damit auch für die Pfahlbaustätten im besonderen. Als Instrumente eines solchen Bildungsauftrages benennt er die Möglichkeiten der Experimentalarchäologie als niederschwelligen Zugang für Kinder und Jugendliche wie insbesondere den Archäologiekoffer mit originalen Objekten, Grabungsbesuche durch Schulklassen und das experimentelle Bauen mit prähistorischen Werkzeugen.

Eva-Maria Seng schildert die Entstehung und Wandlung der Welterbeliste seit den ersten Eintragungen von 1978 und deren anfänglichem Fokus auf Monumenta-

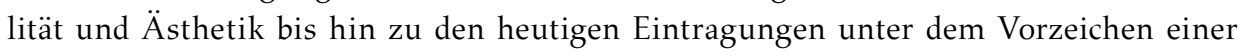
ausbalancierten, repräsentativen, glaubwürdigen Welterbeliste, die zur Berücksichtigung bis dahin unterrepräsentierter Stätten wie Industrielandschaften oder archäologische Stätten führte. Die Erweiterung und Weiterentwicklung umfasste auch das Konzept von seriellen transnationalen Stätten, deren Eigenschaften vor dem Hintergrund der Eintragung der Pfahlbauten ihr besonderes Augenmerk gilt. Die Chance solcherart serieller und transnationaler Stätten sieht sie in der Überwindung heutiger künstlich gesetzter Staatsgrenzen und damit in der Möglichkeit eines Kulturtransfers und Kulturaustausches. Das große Potential der Pfahlbauten liege jedoch nicht mehr in einer additiven Sammlung von Einzelphänomenen, sondern durch die ökologische Zusammenschau von Natur und Kultur unter dem Vorzeichen der Umwelt in einer integralen Sicht auf das Welterbe, das die Verbindung von naturwissenschaftlichen Technologien mit archäologischen, geologischen, geographischen und historischen Methoden zu umwelt- und klimageschichtlichen Erkenntnissen führen könne. Dies gelte es zu vermitteln.

Der Aufsatz von Helmut Schlichtherle widmet sich sowohl der Geschichte der Pfahlbauforschung seit ihren Anfängen, der damit einhergehenden Herausbildung der universitären Wissenschaft der Ur- und Frühgeschichte im Zusammenhang mit den Grabungskampagnen am Bodensee als auch dem Konflikt zwischen Landwirtschaft, Tourismus und Siedlungspolitik aufgrund der daraus resultierenden Trockenlegung von Mooren, Anlage von Jachthäfen und damit der Erosion der Fundstellen. Eindrücklich beschreibt er die Einrichtung der Forschungsstelle in Hemmenhofen am Bodensee mit den dort versammelten Wissenschaftlern und Laboren aus den unterschiedlichsten Disziplinen. Der Autor stellt daraufhin aufgrund der neueren dendrochronologischen, botanischen und textilkundlichen Untersuchungen die Ergebnisse auf dem Gebiet Siedlungsanlagen und Häuser, Wald- und Landwirtschaft der Pfahlbauern, ihrer Ernährung, Krankheiten und Bekleidung vor. Ebenfalls konnten neue Erkenntnisse zur Einführung technischer Innovationen wie dem Rad, dem frühen Fahrzeugbau oder dem Einbaum gewonnen werden. Schließlich können Aussagen über die Dynamik des Siedlungsbaus und der Siedlungsverlagerung wie auch des Kulturpflanzensortiments oder des Kulturtransfers gemacht werden.

Nach diesen drei methodisch-theoretischen Beiträgen zu Bildung, Genese und Verortung der Pfahlbaustätten, deren Potentialen und den Ergebnissen der neueren Pfahl- 
bauforschung folgt mit dem Beitrag Klaus-Dieter Schnells zur Internationalen Bodenseekonferenz die Schilderung der Zusammenarbeit in der Vierländerregion Bodensee im Bereich Umwelt- und Gewässerschutz, Bildung, Kultur, Wirtschaft und Tourismus. Der neuerliche Welterbestatus der Pfahlbauten habe den grenzüberschreitenden Austausch nochmals intensiviert und in eine gemeinsame Strategie zur Vermittlung des Welterbes durch zentrale Anlaufpunkte als Informationszentren zu Pfahlbauten, Schwerpunktmuseen und der Vorstellung von einem Science Center münden lassen.

Der empiriegesättigte Beitrag Anna Michels zu den Potentialen von seriellen und transnationalen Welterbestätten bei der Vermittlungsarbeit kommt in weiten Teilen zu ernüchternden Resultaten hinsichtlich der grenzüberschreitenden Abstimmung, der Zusammenarbeit der verschiedenen Akteure, der unterschiedlichen fachlichen Herkunft der Experten und der damit verbundenen gegenläufigen Ansichten bei zentralen Fragen didaktischer Möglichkeiten und deren Anwendung bei der Vermittlung. Die Heterogenität der Akteure führe zu Interessenkonflikten und verhindere häufig strategische Überlegungen zur Vermittlung. So macht sie gerade an der von der UNESCO propagierten transnationalen Welterbeeintragung und der damit verbundenen Völkerverständigung aufgrund der Schwierigkeiten bei der Zusammenarbeit häufig nur schwer handhabbare Probleme fest.

Die folgenden zwei Beiträge werfen einen Blick auf in einer oder mehrerer Hinsicht vergleichbare serielle Welterbestätten. Jürgen Obmann und Christof Flügel stellen den Obergermanisch-Raetischen Limes vor, die ehemalige römische Reichsgrenze, eine in der Antike durch Wege, Palisaden, Mauern, Wachtürme und Kleinkastelle markierte Zone. Auch der Limes ist heute als archäologische Stätte von Laien nicht leicht wahrzunehmen und damit nur schwer vermittelbar. Der Ruf der Touristiker nach Nach- und Neubauten zur wirtschaftlichen Erschließung, Nutzung und Inwertsetzung führte zu zahlreichen Limes-Turmneubauten, Kastell-Teil-Neubauten und der Errichtung neuer Palisaden, Wall/Graben- und Mauerstücke in höchst unterschiedlicher Form und Ausführung. In den vergangenen Jahren wurden diese Visualisierungsmaßnahmen noch durch spektakuläre Aussichtsplattformen ergänzt wie auch durch Schattenrissfiguren in Stahl oder Kinderspielplätze in Limesanmutung, die so der gewissen Monotonie eines $550 \mathrm{~km}$ langen Denkmals begegnen sollen. Die Alternative dieser problematischen Visualisierungen des Bodendenkmals mit der Errichtung von Museen führe stattdessen häufig zur Verschiebung der Aufmerksamkeit der Besucher hin zum Museum und weg vom Bodendenkmal. Zwar brachte die Eintragung als Welterbe dem Obergermanisch-Raetischen-Limes eine verstärkte Aufmerksamkeit und Auseinandersetzung, jedoch oft nur punktuell auf Einzelelemente reduziert, die einer Sicht auf das gesamte Denkmal entgegenwirken.

Demgegenüber führte - so der Beitrag von Nadine Vivier - die Nominierung und Eintragung des Loire-Tales in Frankreich im Jahr 2000 nach Dammbauten gegen Überschwemmungen und großteils ungeregelten Baumaßnahmen aufgrund der Bevölkerungszunahme in den 1980er Jahren zu einem Neun-Punkte-Managment-Plan von 
2012. Er umfasste Schutz und Entwicklung des Welterbes, die Bewahrung der Landschaft und freie Sicht auf den Fluss, Maßnahmen gegen weitere Zersiedelung, Organisation der Entwicklung der Städte, Integration neuer Einrichtungen, Regelung des Zugangs zu den Stätten des Loiretals, die Entwicklung eines nachhaltigen Tourismus in Einklang mit der Kulturlandschaft, die Stärkung der Werte der Einschreibung und eine Unterstützung und Hilfe für die Verantwortlichen. Zahlreiche Maßnahmen in diesem Sinne wurden mit staatlicher Hilfe und durch EU-Programme umgesetzt. Ebenso wurden entsprechende Vermittlungsprogramme der kulturellen Werte des Loiretals an Kinder, Jugendliche und Erwachsene initiiert und schließlich an den Universitäten der Loire-Region interdisziplinäre Forschungsprogramme zum Kulturerbe und zur Natur und Biodiversität der Flußlandschaft unterstützt. Die serielle UNESCO-Nominierung und Eintragung des Loiretales als positiver und dynamischer Faktor für die Bewahrung der Kulturlandschaft und des Kulturerbes, die Entwicklung der Region unter Einbeziehung der Bevölkerung und der damit verbundenen Bewusstwerdung der Bewohner ihres sie umgebenden Kulturerbes bewertet Vivier abschließend als Erfolg.

Den Bogen zurück zur Vermittlung kulturellen Erbes in unterschiedlichen Institutionen und mit Hilfe unterschiedlicher Medien spannt der nächste Beitrag Tobias Engelsings zur vielbeachteten Präsentation der prähistorischen Objekte in Konstanz im 19. Jahrhundert. Die Geschichte um den Altertumsforscher, Botaniker und Museumsgründer Ludwig Leiner schildert die Anfangszeit der Pfahlbaufunde am Bodensee und damit das einsetzende Pfahlbaufieber. Die Pfahlbausammlungen bildeten denn auch die Hauptattraktion des Museums für Wissenschaftler, Forscher, hochgestellte Persönlichkeiten und Museumsfachleute wie für den Gründer des Germanischen Nationalmuseums Hans Freiherr von und zu Aufseß oder den Pathologen, Prähistoriker und Gründer des Berliner Völkerkundemuseums Rudolf Virchow. Den Mittelpunkt des Rosgartenmuseums in Konstanz und damit auch des Museumsbesuchs bildet der bis heute original erhaltene "Leinersaal" mit den damals angefertigten Vitrinen, mit der bis heute erhaltenen Präsentation der Fundstücke durch Leiner sowie den Ölgemälden Anton Seders von 1876.

Michael Herdeck widmet sich dann den unterschiedlichen Formen der experimentellen Archäologie, ausgehend vom Pfahlbaumuseum in Unteruhldingen, wie auch des weiteren der Reenactment-Bewegung insbesondere im angelsächsischen und skandinavischen Raum und den damit verbundenen Gefahren und Chancen. Anhand zahlreicher Beispiele werden Potentiale und Nachteile für Vermittlung und wissenschaftliches Experiment vorgestellt sowie insbesondere die Notwendigkeit schriftlicher Überlieferung, die jedoch bei den Pfahlbauten nicht gegeben ist. Hier plädiert Herdeck stattdessen für archäotechnische Demonstrationen.

Dem Verhältnis von Archäologen und Filmemachern zur Vermittlung von wissenschaftlichen Erkenntnissen an ein breites Publikum ist der Beitrag von Elisabeth Milin geschuldet. Sie beschreibt und analysiert den Paradigmenwechsel des öffentlich-rechtlichen Fernsehens unter dem Druck der privaten Sender, und zwar weg vom Auftrag des öffentlichen Fernsehens als Instrument der Aufklärung hin zu einem Dienstleister der 
Zuschauer. Damit einher ging der Wandel des Dokumentarfilms vom Informieren zum Erzählen, in dessen Verlauf der Archäologe häufig zum Helden und seine Grabung zur Kriminalstory mutiert.

Das Buch beschließt ein Beitrag zum Welterbe-Tourismus von Kurt Luger. Er analysiert und diskutiert darin an einschlägigen Fällen den Zielkonflikt zwischen kulturellem Erbe und touristischer Vermarktung, dessen Lösung er in einem qualitätsorientierten Kulturtourismus unter Beachtung und Einbindung von Nachhaltigkeitskriterien sieht.

Der Tagungsband zum unsichtbaren Welterbe der Prähistorischen Pfahlbauten im Alpenraum und deren Erschließung und Vermittlung versucht ausgehend von Geschichte, Erforschung, Präsentation und Vermittlung der Pfahlbauten sowohl die unterschiedlichen Strategien, Probleme und Möglichkeiten des komplexen Welterbes aufzuzeigen als auch mit weiteren seriellen Welterbestätten zu vergleichen. Die unterschiedlichen methodischen, theoretischen und empirischen Ansätze der versammelten Beiträge sollen zum weiteren Diskurs um die Transmission von kulturellem Erbe an eine interessierte Öffentlichkeit beitragen. 